\title{
Relationship of the hierarchy of dominance and abundance in the structure of the ant-plant interaction network
}

\section{Relación de la jerarquía de dominancia y abundancia en la estructura de la red de interacción planta-hormiga}

Brenda Juárez-Juárez ${ }^{1}$, Mariana Cuautle², Jorge E. Valenzuela-González ${ }^{3}$, Citlalli CastilloGuevara $^{4 *}$

${ }^{1}$ Maestría en Biotecnología y Manejo de Recursos Naturales, Universidad Autónoma de Tlaxcala, Ixtacuixtla, Tlaxcala, México.

${ }^{2}$ Departamento de Ciencias Químico-Biológicas, Universidad de las Américas Puebla (UDLAP), San Andrés Cholula, Puebla, México.

${ }^{3}$ Departamento de Ecología Funcional. Instituto de Ecología A.C, Xalapa, México.

${ }^{4}$ Centro de Investigación en Ciencias Biológicas, Universidad Autónoma de Tlaxcala, Ixtacuixtla, Tlaxcala, México, guevaracc@yahoo.com.mx.

https://doi.org/10.29267/mxjb.2017.2.1.67

\begin{abstract}
Ants, like other organisms, have a hierarchy of dominance where each individual establishes a range that determines their access to resources. In ant-plant interactions, competitively superior and territorial ant species can limit access to the resource for submissive species. Some studies mention that abundance contributes to the organization of these complex networks of interaction. However, abundance outside the ecological network is only partially explained by the probability of ant species finding their food resource. Despite the importance of competition within the structure of the ant-plant interaction network, few studies have been done on the dominance hierarchy behavior, which could help us to understand the structural complexity of the ant community in different types of ecosystems. In this article we will make a brief review of how the hierarchical behavior of ants and their abundance can determine the structure of the ant-plant interaction network.
\end{abstract}

Keywords: abundance, ant-plant interaction, competition, food resource, hierarchy of dominance, network structure.

\section{RESUMEN}

Las hormigas al igual que otros organismos presentan una jerarquía de dominancia en donde cada individuo establece un rango que determina su acceso a los recursos. En las interacciones planta-hormiga, las especies de hormigas competitivamente superiores y 
territoriales pueden limitar el acceso al recurso a las especies sumisas. Algunos estudios mencionan que la abundancia contribuye a la organización de estas complejas redes de interacción. Sin embargo, se considera que la abundancia fuera de la red ecológica sólo explica parcialmente la probabilidad de que las especies de hormigas encuentren su recurso alimenticio. A pesar de la importancia de la competencia dentro de la estructura de la red de interacción planta-hormiga, se han hecho pocos estudios en cuanto al comportamiento de jerarquía de dominancia, lo cual nos podría ayudar a entender la complejidad estructural de la comunidad de hormigas en los diferentes tipos de ecosistemas. En este artículo haremos una breve revisión de como el comportamiento jerárquico de las hormigas y su abundancia pueden determinar la estructura de la red de interacción planta-hormiga.

Palabras clave: abundancia, competencia, estructura de la red, interacción planta-hormiga, jerarquía de dominancia, recurso alimenticio.

\section{INTRODUCCIÓN}

Las hormigas (Hymenoptera: Formicidae) son organismos dominantes en la mayoría de los hábitats terrestres, habiendo alcanzado, entre los insectos sociales, la radiación adaptativa más impresionante. La clave de su éxito es su vida colonial; la complejidad de su vida social permite a las hormigas controlar su entorno físico, explotar los recursos de una manera eficiente y superar a los competidores (Hölldobler \& Wilson, 2009). Se les considera organismos eusociales o verdaderamente sociales porque presentan cuidado cooperativo de la descendencia, generaciones que se sobrelapan y un sistema de castas altamente organizado (Wilson, 1971). Las castas son grupos especializados dentro de la colonia que realizan diferentes funciones y tienen modificaciones morfológicas (Kaspari, 2000).

Dentro de la macrofauna edáfica, las hormigas son siempre uno de los grupos más abundantes y diversos, en ecosistemas naturales y agroecosistemas (Lavelle \& Kohlman, 1984; Camacho, 1995; Delabie \& Fowler, 1995). En estos sistemas desempeñan un papel muy importante como depredadoras, herbívoras o detritívoras, y participan en los procesos fisicoquímicos del suelo, incluyendo la descomposición y el reciclaje de nutrientes (Lobry de Bruyn \& Conacher, 1990; Brussard et al., 1997).

Las hormigas se distribuyen virtualmente en todos los hábitats, estableciendo interacciones con las plantas que resultan fundamentales en la naturaleza (Beattie \& Hughes, 2009). Utilizan hojas, tallos, flores, frutos y semillas, lo que da lugar a una enorme diversidad de interacciones. Defensa frente a herbívoros, dispersión de semillas, polinización, depredación de semillas, herbivoría, e incluso la formación de auténticos jardines de epífitas, son algunas de las principales actividades desarrolladas por las hormigas (RicoGray \& Oliveira, 2007). La influencia de las hormigas sobre las características fisicoquímicas del suelo llega a repercutir en última instancia sobre el establecimiento, abundancia o composición de muchas especies vegetales (Culver \& Beattie, 1983; Folgarait et al., 2002; Wagner \& Nicklen, 2010). Además, muchas especies de hormigas pueden utilizar una sustancia azucarada excretada por hemípteros ("rocío de miel") como la 
principal fuente de carbohidratos en su dieta, estas hormigas protegen a los hemípteros y sus plantas huésped de enemigos naturales (Buckley, 1987; Cushman \& Whitham, 1991).

En este artículo haremos una breve revisión de como el comportamiento jerárquico de las hormigas y su abundancia pueden determinar la estructura de la red de interacción plantahormiga (Fig. 1). Para ello se enlistan los tipos de sistemas jerárquicos en las hormigas y su relación con la competencia, se describirán las características de la red de interacción planta-hormiga $\mathrm{y}$, finalmente se analizarán estudios que relacionan la jerarquía de dominancia y la abundancia en la estructura de la red de interacción planta-hormiga.

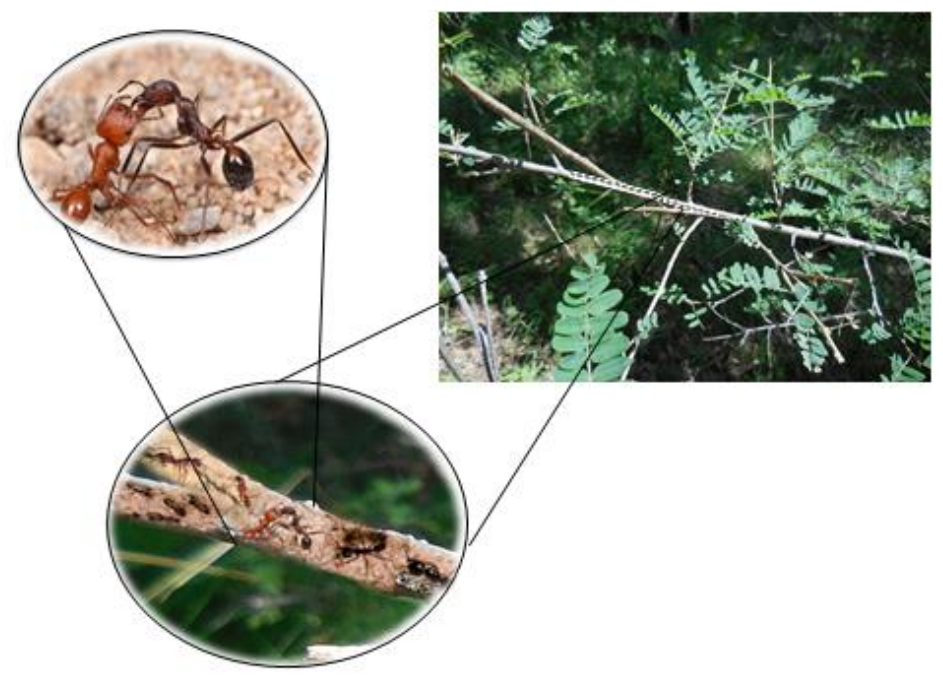

Fig. 1. Relación de la jerarquía de dominancia y abundancia en la estructura de la red de interacción planta-hormiga.

\subsection{Sistema jerárquico y la competencia en las hormigas}

En una jerarquía de dominancia cada individuo establece un rango que determina su acceso a los recursos. Las comunidades de hormigas suelen estar estructuradas en jerarquías de dominancia (Fellers, 1987; Parr \& Gibb, 2010), las especies dominantes desplazan agresivamente a los subordinados de los recursos.

En muchas comunidades de hormigas de zonas templadas hay una aparente jerarquía regular (Wilson, 1971; Savolainen \& Vepsäläinen, 1988; Savolainen, 1990; Andersen, 1992). Estas jerarquías se han generalizado de varias formas pero se reducen a dominantes y subordinadas. Las dominantes frecuentemente forman grandes colonias, presentan un comportamiento territorial agresivo y reclutamiento rápido del alimento. La diversidad y densidad de hormigas es frecuentemente baja alrededor de las especies dominantes. Las especies subordinadas frecuentemente forman colonias pequeñas, con baja capacidad de reclutamiento y se encuentran en la periferia de los territorios controlados por las dominantes. Este patrón es excepcionalmente pronunciado en los hábitats templados y boreales del norte, en los áridos desiertos y matorrales en Australia, y en las comunidades simples (Hölldobler \& Wilson, 1990). 
Esta jerarquía de dominancia no es en absoluto universal en las comunidades de hormigas. Las hormigas de la copa tropical, por ejemplo, forman "islas", con troncos estrechos y defendibles que conducen a la exuberante vegetación de arriba (Hölldobler \& Lumsden, 1980). Una sola gran colonia puede monopolizar árboles enteros y evitar otras especies (Hölldobler, 1983; Adams, 1994). Fenómenos similares, a escalas más pequeñas, ocurren en las hormigas de las plantas del sotobosque tropical (Davidson et al., 1989).

Un factor importante en el ensamble de las comunidades de hormigas es la competencia, que puede presentarse entre organismos de una especie (intraespecífica) o entre las especies (interespecíficas). La competencia se puede producir de manera indirecta, a través del consumo de alimentos u otros recursos, o bien directamente, a través de la muerte de otras colonias. Donde hay abundante radiación solar, existe una creciente evidencia de que las colonias de hormigas compiten entre sí por los recursos, tales como nidos y alimentos. Muchas especies de hormigas son muy territoriales. Si las colonias agotan los recursos y matan a las fundadoras cerca de nidos establecidos, esto debería resultar en una distribución regular de las colonias de hormigas en un paisaje homogéneo (Kaspari, 2000).

La competencia intra e interespecífica entre las hormigas es considerada uno de los principales mecanismos de estructuración de ensamblaje de hormigas locales (Andersen, 1992; Parr et al., 2005; Hölldobler \& Wilson, 2008). Este fuerte papel de la competencia posiblemente se debe a que la mayoría de las especies de hormigas son forrajeras del lugar central y tienen requerimientos similares (sitios de anidación y suministros de alimento), lo que resulta en una menor superposición de sus áreas de alimentación (López et al., 1994).

En las hormigas, la competencia interespecífica es particularmente pronunciada (Parr \& Gibb, 2012), ya que "el peor enemigo de una hormiga es otra hormiga" (Forel, 1874). Por lo tanto, la información sobre la afiliación taxonómica de un oponente puede ser de gran importancia para una especie de hormiga. Sin embargo, la competencia interespecífica en especies sociales y especialmente en hormigas puede depender no sólo de las respectivas especies presentes en un sitio, sino también de su abundancia relativa (Helfman, 1989).

Por otro lado, diversos estudios han mostrado una clara correlación positiva entre la posición en una jerarquía competitiva y el grado de termofilia de las especies (Andersen, 1995; Bestelmeyer, 2000). Sin embargo, esto contrasta con los resultados obtenidos para las comunidades de hormigas mediterráneas (Cerdá et al., 1998; Retana \& Cerdá, 2000): las especies subordinadas son las más termófilas y mejores adaptadas a la parte más cálida del día, mientras que las especies conductualmente dominantes son más activas durante menos horas de calor en los hábitats soleados abiertos. Estos estudios también demuestran que las variaciones diarias de temperatura pueden interrumpir las jerarquías competitivas lineales y conducir a diferentes patrones de dominancia en diferentes momentos del día. Por otro lado, otros estudios mencionan que las especies dominantes y subordinadas tienen patrones de forrajeo similares en relación con la temperatura (Andersen, 1992; LeBrun, 2005).

Para contrarrestar los costos de acondicionamiento físico de ser superados, los subordinados necesitan estrategias alternativas para poder co-ocurrir con dominantes. Además de la diferenciación de nichos, los subordinados a menudo explotan las señales visuales (Hunter et al., 2007), acústicas (Durant, 2000) o vibracionales (Evans et al., 2009) para detectar y evitar competidores dominantes. Sin embargo, las señales indirectas, como 
las huellas químicas no intencionadamente dejadas, tienen la ventaja de que son más persistentes que las señales directas y, por tanto, pueden indicar la presencia previa de competidores (Kats \& Dill, 1998).

Al igual que los depredadores de caza olfativos que usan señales químicas para detectar y localizar a sus presas (Hughes et al., 2010), las especies dominantes también podrían buscar subordinados a través de señales olfativas, ya sea para atacarlas (Carthey et al., 2011) para robar su alimento (cleptoparasitismo: Creel et al., 2001; Nieh et al., 2004) o desplazarlas de su territorio. Las señales olfativas lanzadas por los subordinados y/o competidores dominantes podrían influir en la competencia interespecífica y, en consecuencia, afectar la dinámica de la comunidad.

Debido a su diverso sistema de comunicación química, las hormigas son ideales para investigar el papel de las señales químicas en la competencia interespecífica (Hölldobler \& Wilson, 1990). Estos insectos sociales usan hidrocarburos cuticulares para distinguir a sus compañeros de no-nidos, pero también pueden reconocer otras especies basadas en sus perfiles químicos (Dresche et al., 2011; Lang \& Menzel, 2011), que especies son, y con frecuencia incluso de una casta específica (Howard et al., 1982; Bagnéres et al., 1998,). Al caminar sobre superficies receptivas, los insectos dejan accidentalmente huellas químicas, que son congruentes con el perfil cuticular del individuo (Akino \& Yamaoka, 2005; Devigne \& De Biseau, 2012) y que pueden ser detectados por otros (Eltz, 2006).

Fellers (1987), trabajando con un ensamblaje de hormigas del bosque, sugirió un equilibrio evolutivo entre la capacidad de descubrimiento de recursos (encontrar y usar recursos antes que los competidores) y la dominación conductual de los recursos. La existencia de tal compensación puede tener importantes efectos en la estructura y funcionalidad de las asociaciones de hormigas, ya que permitiría la coexistencia de especies que abarcan el rango de expresión de rasgos negativamente correlacionados (Kneitel \& Chase, 2004). Davidson (1998), sin embargo, postuló que algunas especies pueden romper este intercambio de dominación/descubrimiento a través de su capacidad para ser el mejor en ambos lados; además de discutir que las características de estos taxa podrían haber facilitado la evolución del dominio conductual y numérico (Holway, 1999).

\section{RED DE INTERACCIÓN PLANTA-HORMIGA}

En una comunidad cada especie interactúa de diferente manera con una o varias especies formando complejas redes de especies (Jordano, 1987). Una red de especies interactuantes puede tener un número reducido de enlaces entre las especies, indicando un ensamble de especialistas ecológicos, o muchos enlaces, indicando generalistas ecológicos (Bascompte \& Jordano, 2006).

Las interacciones mutualistas constituyen la base para el funcionamiento de muchos ecosistemas, dado que de ellas depende gran parte de la reproducción y reclutamiento exitoso de muchas especies de plantas. Además, gran número de especies animales dependen estrechamente de los recursos que las plantas proveen alrededor de sus estructuras reproductivas (flores, frutos, etc.). Por lo tanto, estas interacciones sustentan la 
biodiversidad y funcionalidad de las comunidades naturales y han tenido un papel central en la evolución de la biodiversidad terrestre (Thompson, 2006).

Una red de interacciones es un conjunto de dos tipos de elementos: nodos o vértices y los enlaces entre ellos. Además, pueden existir otras estructuras como subredes o cliques, que son subconjuntos definidos de nodos. Existen dos tipos diferentes de redes que pueden representarse con distintos tipos de grafos: redes unipartitas o uni-modales (one-mode networks), ya que los enlaces se pueden establecer entre cualquiera de los nodos de la red, y redes bipartitas o bi-modales (two-mode networks). Ambas, a su vez, pueden ser redes no dirigidas (o simples) y redes dirigidas. Las redes no dirigidas y dirigidas sólo difieren por el hecho de que estas últimas contienen información del flujo y dirección de la interacción. Por ejemplo, la mayor parte de las redes tróficas se pueden caracterizarse por la dirección de transferencia de energía (de presa a depredador). Las redes bipartitas ilustran las conexiones entre nodos de dos grupos distintos, de tal modo que no existen conexiones entre nodos del mismo grupo (Borgatti \& Everett, 1997; Newman et al., 2006).

Por otra parte, cualquiera de estas redes puede ser una red cuantitativa o ponderada si, además de la información sobre presencia-ausencia de los enlaces, la red contiene información cuantitativa sobre la intensidad de los enlaces (Newman, 2004).

Las interacciones entre hormigas y plantas constituyen ejemplos que van desde el antagonismo y el oportunismo hasta el mutualismo ocasional y la simbiosis obligada. Además, estas interacciones están geográficamente extendidas y han demostrado ser cruciales en la conformación de muchos ecosistemas (Höldobler \& Wilson, 1990, 2009; Rico-Gray \& Oliveira, 2007).

Las interacciones planta-hormiga que se consideran mutualismos altamente evolucionados son las que se han estudiado más en detalle. Entre estos destacan los sistemas en los que hormigas protegen a las plantas de los herbívoros a cambio de una recompensa que puede ser bien domacios (cavidades producidas por las plantas a partir de diferentes órganos, en las que las hormigas pueden anidar) y/o bien alimento proporcionado por el néctar extrafloral o los corpúsculos nutritivos (Janzen, 1966; Heil \& McKey, 2003).

Las hormigas mutualistas se consideran generalmente como una herramienta eficaz para la defensa indirecta de las plantas contra herbívoros (Heil \& McKey, 2003). Varias características de la ecología de la alimentación y el comportamiento social de las hormigas las convierten en el compañero ideal para fines de defensa: construyen nidos estables, patrullan amplias áreas día y noche, defienden territorios y a menudo pueden adoptar estrategias de reclutamiento eficaces hacia el lugar donde se encuentran abundantes alimentos o amenazas potenciales (Hölldobler \& Wilson, 1990). Además, varias especies de hormigas son colectores de azúcar y depredadores robustos, exhibiendo reacciones muy agresivas contra otros animales que pueden representar una amenaza potencial para sus recursos alimenticios y sitios de anidación (Rico-Gray \& Oliveira, 2007).

Con el fin de explotar estos servicios y mejorar la calidad y estabilidad de la asociación, las plantas necesitan atraer a las hormigas proporcionándoles refugios y fuentes de alimentos ricos en nutrientes. Además, la evidencia también indica que los nectarios extraflorales 
(NEF) puede aumentar significativamente la supervivencia, el crecimiento y la reproducción de colonia de hormigas (Byk \& Del-Claro, 2011).

\section{JERARQUÍA DE DOMINANCIA Y ABUNDANCIA EN LA ESTRUCTURA DE LA RED DE INTERACCIÓN PLANTA-HORMIGA}

La relación entre descubrimiento y dominación es la relación inversa entre la capacidad de una especie para descubrir un recurso y el dominio de las especies de ese recurso. Para las hormigas, la compensación del descubrimiento-dominación primero fue descrita por Fellers (1987) que trabajaba en un ensamblaje de hormigas del arbolado. Esta compensación es ampliamente citada en la literatura sobre ecología de las hormigas y, por consiguiente, ha recibido aceptación dentro de la literatura (Davidson, 1998; Feener 2000; Adler et al., 2007; Delsinne et al., 2007; Pearce-Duvet \& Feener, 2010).

Por otro lado, Davidson (1998) sugirió que el equilibrio entre el descubrimiento y la dominación se rompe por especies que tienen capacidades mejoradas para acceder a los recursos de carbohidratos ("dominantes ecológicos" a menudo especies territoriales) y también por especies invasoras, que han escapado a sus enemigos naturales o limitaciones (Feener, 2000).

La estructura del hábitat también puede influir en la relación de descubrimiento y dominancia porque se ha demostrado que afecta la capacidad de las hormigas trabajadoras para localizar y capturar los recursos alimenticios (Parr \& Gib, 2010).

Uno de los principales objetivos de la ecología es identificar cómo interactúan entre sí diferentes especies y cómo estas interacciones persisten en el espacio y el tiempo (Ings et al., 2009). Estudios recientes han utilizado herramientas derivadas de la teoría de redes para investigar la organización compleja de estas interacciones a nivel de comunidades ecológicas (Hagen et al., 2012). Bajo este marco unificado de interacciones entre especies, los estudios se han centrado en las propiedades estructurales de estas redes en diferentes sistemas y hábitats a lo largo de la Tierra (Olesen \& Jordano, 2002).

Por otra parte, algunos estudios han puesto de relieve diferentes factores que contribuyen a la organización de estas complejas redes de interacción (por ejemplo, abundancia, filogenia, riqueza de especies, etc.,) (Medan et al., 2007; Rezende et al., 2007). Aunque el conocimiento sobre la organización de las redes ecológicas ha aumentado en muchos sistemas de estudio (por ejemplo, plantas-polinizadores y dispersores de plantas) (Memmott et al., 2007; Santamaría \& Rodríguez-Gironés, 2007) factores que contribuyen a la estructuración de redes de interacción que involucran hormigas y plantas con nectarios extraflorales (plantas con NEF) (Chamberlain et al., 2010; Lange et al., 2013). En estos sistemas, las plantas secretan un líquido rico en carbohidratos (predominantemente sacarosa, glucosa y fructosa), aminoácidos y otros compuestos que atraen y benefician a las hormigas (Byk \& Del-Claro, 2011).

Las redes ecológicas que involucran hormigas y plantas portadoras de NEF muestran un patrón altamente anidado (Guimarães et al., 2006; Rico-Gray et al., 2012; Dáttilo et al., 2013a). Esto indica que dentro de una red de hormigas, un núcleo central de especies tiene 
muchas interacciones entre sí y especies periféricas con pocas interacciones interactúan con un subconjunto adecuado del núcleo central de los generalistas con mayor interacción (Bascompte et al., 2003; Guimarães et al., 2006).

Estudios recientes se han centrado en la dinámica evolutiva del núcleo central de las especies de hormigas generalistas en las redes de hormigas (Lange et al., 2013; Dáttilo et al., 2013a; Dáttilo et al., 2013b). Estos estudios muestran que las especies de hormigas presentes en el núcleo central interactúan con las plantas más de lo esperado por sus abundancias naturales en la vegetación (Dáttilo et al., 2014a). Sin embargo se considera que la abundancia espacial fuera de la red ecológica sólo explica parcialmente la probabilidad de que estas especies de hormigas generalistas encuentren su recurso alimenticio (Dáttilo et al., 2014a). Además, la composición de las hormigas en el núcleo central puede ser estable a lo largo de grandes distancias geográficas, fases fenológicas de la secreción de néctar o incluso después de perturbaciones causadas por huracanes tropicales (Sánchez-Galván et al., 2012; Dáttilo et al., 2013a). Sobre la base de la estabilidad de estas especies de hormigas centrales en la monopolización del recurso, podemos esperar que estas hormigas puedan ser competitivamente superiores (es decir, por reclutamiento masivo y/o comportamiento agresivo) en comparación con las otras especies encontradas en la red (Dáttilo et al., 2013b, 2014b, 2014c).

En las interacciones hormiga-planta, las especies de hormigas competitivamente superiores y territoriales pueden limitar el acceso al recurso por las especies sumisas debido principalmente al hecho de que el néctar es un recurso altamente nutritivo y predecible (Heil \& McKey, 2003; Blüthgen \& Fiedler, 2004a) basándose en la importancia de la competencia para la estructura de la comunidad de hormigas, podemos esperar que sea también una fuerza muy importante que moldee la estructura de la red ecológica.

Los estudios teóricos basados en el modelo de preferencias compartidas (Svanbäck \& Bolnick, 2005) sugieren que la competencia puede conducir a un patrón anidado en redes basadas en individuos (Araújo et al., 2010; Pires et al., 2011). Por ejemplo, si individuos con capacidades competitivas diferentes exhiben idénticas preferencias por los diferentes recursos alimenticios, las dietas de individuos más competitivos representan subconjuntos de las dietas de otros individuos menos competitivos, generando nidos (Araújo et al., 2010; Tinker et al., 2012).

Aunque la competencia ha sido ampliamente ignorada en los estudios de redes mutualistas que involucran hormigas y plantas portadoras de NEF (Chamberlain \& Holland, 2009; Dáttilo et al., 2014c), la jerarquía de dominancia juega un papel importante en la estructuración del patrón anidado en las redes de hormigas, en donde las hormigas que se encuentran en el núcleo central de los generalistas son competitivamente superiores a las especies de hormigas periféricas y sumisas (Dattilo et al., 2014).

\section{CONCLUSIONES}

El estudio de las interacciones mutualistas entre plantas y animales es importante, tanto para comprender los sistemas ecológicos como para poder emplear métodos para un buen manejo y conservación (Bronstein et al., 2006; Rico-Gray \& Oliveira, 2007). Las hormigas 
establecen relaciones mutualistas con las plantas y lo que se sabe es que generalmente se trata de redes anidadas, por lo que es importante resaltar su importancia para estudiarlas a profundidad ya que pueden ser altamente vulnerables a la extinción de especies por la fuerza que presentan las interacciones de este tipo de redes. En cuanto al comportamiento de la jerarquía de dominancia y abundancia dentro de la red de interacción planta-hormiga son pocos los estudios que se han realizado y se han realizado en zonas tropicales, por lo que sería conveniente hacer más estudios en otros tipos de ambientes. Esto también nos ayudará a comprender de una mejor manera como es que se estructuran las comunidades de las hormigas en los diferentes tipos de ecosistemas.

\section{REFERENCIAS}

Adams E. S. 1994. Territory defense by the ant Azteca trigona: Maintenance of an arboreal ant mosaic. Oecologia. 97: 202-208.

Adler R. F., LeBrun E. G. \& Feener Jr, D. H. 2007. Maintaining diversity in an ant community: modeling, extending and testing the dominance-discovery trade-off. The American Naturalist. 169: 323-333.

Akino T. \& Yamaoka R. 2005. Trail discrimination signal of Lasius japonicas (Hymenoptera: Formicidae). Chemoecology. 15(1): 21-30.

Andersen A. N. 1992. Regulation of momentary diversity by dominant species in exceptionally rich ant communities of the Australian seasonal tropics. American Naturalist. 140: 401-420.

Andersen A. N. 1995. A classification of Australian ant communities based on functional groups which parallel plant life-forms in relation to stress and disturbance. Journal of Biogeography. 20: 15-29.

Araújo M. S., Martins E. G., Cruz L. D., Fernandes F. L., Linhares A. X., Dos Reis S. F. \& Guimaraes P. R. 2010. Nested diets: a novel pattern of individual-level resource use. Oikos. 119: 81-88.

Arnan X., Cerda X., Rodrigo A. \& Retana J. 2013. Response of ant functional composition to fire. Ecography. 36: 1182-1192.

Bagnéres A. G., Riviere G. \& Clement J. 1998. Artificial neural network modeling of caste odor discrimination based on cuticular hydrocarbons in termites. Chemoecology. 8: 201209.

Bascompte J. \& Jordano P. 2006. The structure of plant animal mutualistic networks. En: M. Pascual \& J.A. Dunne (eds.), Food Webs as Complex Adaptative Networks: liking structure to dyamics. Oxford University Press, Oxford. Pp. 143-159. 
Bascompte J., Jordano P., Melián C. J. \& Olesen J. M. 2003. The nested assembly of plantanimal mutualistic networks. Proceedings of the National Academy of Sciences of the United States of America. 100: 9383-9387.

Beattie A. J. \& Hughes L. 2009. Ant-plant interactions. En: Herrera, C.M., Pellmyr, O. (eds.), Plant-animal interactions: an evolutionary approach, pp. 211-247. WileyBlackwell, Hoboken, NJ, Estados Unidos.

Bestelmeyer B. T. 2000. The trade-off between thermal tolerance and behavioural dominance in a subtropical South American ant community. Journal of Animal Ecology. 69: 998-1009.

Blüthgen N. \& Fiedler K. 2004a. Competition for composition: lessons from nectar-feeding ant communities. Ecology. 85: 1479-1485.

Byk J. \& Del-Claro K. 2011. Ant-plant interaction in the Neotropical savanna: direct beneficial effects of extrafloral nectar on ant colony fitness. Population Ecology. 53: 327332.

Borgatti S. P. \& Everett M. G. 1997. Network analysis of 2-mode data. Social Networks. 19:243-69.

Brusaard L., Behan-Plletier V., Bignell D., Brown V., Didden W., Folgarait P., Fragoso C., Freckman D., Gupta V. S. R., Hattori S. T., Hawksworth D. L., Klopatek C., Lavelle P., Malloch D., Rusek J., Söderström B., Tiedje J \& Virginia R. 1997. Biodiversity and Ecosystem functioning in Soil. Ambio. 26: 563-570.

Buckley R. 1987. Interactions involving plants, Homoptera, and ants. Annual Review of Ecology, Evolution, and Systematics. 18: 111-138.

Bronstein J. L., Alarcón R. \& Geber M. 2006. Tansley review: evolution of insect/plant mutualisms. New Phytologist. 172: 412-428.

Byk J. \& Del-Claro K. 2011. Ant-plant interaction in the Neotropical savanna: direct beneficial effects of extrafloral nectar on ant colony fitness. Population Ecology. 53: 327 332.

Camacho G. 1995. Estudio de la macrofauna edáfica de 3 agroecosistemas en La Mancha, Ver. Tesis Profesional. Facultad de Biología. Universidad Veracruzana. 63 pp.

Carthey A. J. R., Bytheway J. P. \& Banks P. B. 2011. Negotiating a noisy, information-rich environment in search of cryptic prey: olfactory predators need patchiness in prey cues. Journal of Animal Ecology. 80(4): 742-752. 
Cerdá X., Retana J. \& Manzaneda J. 1998. The role of competition by dominants and temperature in the foraging of subordinate species in Mediterranean ant communities. Oecologia. 117: $404-412$.

Chamberlain S. A. \& Holland J. N. 2009. Body size predicts degree in ant-plant mutualistic networks. Functional Ecology. 23: 196-202.

Chamberlain S. A., Kilpatrick J. R. \& Holland J. N. 2010. Do extrafloral nectar resources, species abundances, and body sizes contribute to the structure of ant-plant mutualistic networks? Oecologia. 164: 741-750.

Creel S., Sprong G. \& Creel N. 2001. Interspecific competition and the population biology of extinction-prone carnivores. In J. L. Gittleman, S. M. Funk, D. Macdonald, \& R. K. Wayne (Eds.), Carnivore conservation. Cambridge, U.K.: Cambridge University Press.

Culver D. C. \& Beattie A. J. 1983. Effects of ant mounds on soil chemistry and vegetation patterns in a Colorado montane meadow. Ecology. 64: 485-492.

Cushman J. H \& Whitham T. G. 1991. Competition mediating the outcome of a mutualism: protective services of ants as a limiting resource for membracids. American Naturalist. 138: 851-865.

Dáttilo W., Rico-Gray V., Rodrigues D. J. \& Izzo T. J. 2013a. Soil and vegetation features determine the nested pattern of ant-plant networks in a tropical rainforest. Ecological Entomology. 38: 374-380.

Dáttilo W., Guimarães P. R. \& Izzo T. J. 2013b. Spatial structure of ant-plant mutualistic networks. Oikos. 122: 1643- 1648.

Dáttilo W., Marquitti F. M. D., Guimarães P. R. \& Izzo T. J. 2014a. The structure of antplant ecological networks: is abundance enough? Ecology. 95: 475-485.

Dáttilo W., Sánchez-Galván I., Lange D., Del-Claro K. \& Rico-Gray V. 2014b. Importance of interaction frequency in analysis of ant-plant networks in tropical environments. Journal of Tropical Ecology. 30: 165-168.

Dáttilo W., Fagundes R., Gurka C. A. Q., Silva M. S. A., Vieira M. C. L., Izzo T. J., DíazCastelazo C., Del-Claro K. \& Rico-Gray V. 2014c. Individual-based ant-plant networks: diurnal-nocturnal structure and species-area relationship. PLOS ONE. 9: e99838.

Dáttilo W., Diaz-Castelazo C. \& Rico-Gray V. 2014. Ant dominance hierarchy determines the nested pattern in ant-plant networks. Biological Journal of the Linnean Society. 10: 153. 
Davidson D. W. 1998. Resource discovery versus resource domination in ants: A functional mechanism for breaking the tradeoff. Ecological Entomology. 23: 484-490.

Davidson D. W., Snelling R.R \& Longino J. T. 1989. Competition among ants for myrmecophytes and the significance of plant trichomes. Biotropica. 21: 64-73.

Delabie J. H. C \& Fowler H. G. 1995. Soil and litter cryptic ant assemblages of Bahian cocoa plantations. Pedobiologia. 39: 423-433.

Devigne C. \& De Biseau J. C. 2012. The differential response of workers and queens of the ant Lasius niger to an environment marked by workers: ants dislike the unknown. Behavioural Processes. 91(3): 275-281.

Drescher J., Feldhaar H. \& Blüthgen N. 2011. Interspecific aggression and resource monopolization of the invasive ant Anoplolepis gracilipes in Malaysian Borneo. Biotropica. 43(1): 93-99.

Durant S. M. 2000. Living with the enemy: avoidance of hyenas and lions by cheetahs in the Serengeti. Behavioral Ecology, 11(6), 624-632. Eltz T. 2006. Tracing pollinator footprints on natural flowers. Journal of Chemical Ecology. 32(5): 907-915.

Eltz T. 2006. Tracing pollinator footprints on natural flowers. Journal of Chemical Ecology. 32(5): 907-915.

Evans T. A., Inta R., Lai J. C. S., Prueger S., Foo N. W., Fu E. \& W. 2009. Termites eavesdrop to avoid competitors. Proceedings of the Royal Society B: Biological Sciences. 276(1675): 4035-4041.

Fellers J. H. 1987. Interference and exploitation in a guild of woodland ants. Ecological Society of America. 68(5): 1466-1478.

Feener Jr D. H. 2000. Is the assembly of ant communities mediated by parasitoids? Oikos. 90: 79-88.

Folgarait P. J., Perelman S., Gorosito N., Pizzio R. \& Fernández J. 2002. Effects of Camponotus punctulatus ants on plant community composition and soil properties across land-use histories. Plant Ecology. 163: 1-13.

Forel A. 1874. Les fourmis de la Suisse. Zurich, Switzerland: Société Helvétique des Sciences Naturelles.

Guimarães P. R., Rico-Gray V., Dos-Reis S. F. \& Thompson J. N. 2006. Asymmetries in specialization in ant-plant mutualistic networks. Proceedings of the Royal Society of London B. 273: 2041-2047. 
Hagen M., Kissiling W. D., Rasmussen C., de Aguiar M. A. M., Brown L., Carstensen D. W., Alves-dos-Santos I., Dupont Y. L., Edwards F. K., Genini J., Guimarães P. R., Jenkins G. B., Jordano P., Kaiser-Bunbury C. N., Ledger M., Maia K. P., Marquitti F. M. D., McLaughlin O., Morellato L. P. C., O’Gorman E. J., Trojelsgaard K., Tylianakis J. M., Vidal M. M., Woodward G. \& Olesen J. 2012. Biodiversity, species interactions and ecological networks in a fragmented world. Advances in Ecological Research. 46: 89-210.

Heil M. \& McKey D. 2003. Protective ant-plant interactions as model systems in ecological and evolutionary research. Annual Review of Ecology, Evolution and Systematics. 34: 425-453.

Helfman G. S. 1989. Threat-sensitive predator avoidance in damselfish-trumpetfish interactions. Behavioral Ecology and Sociobiology. 24(1): 47-58.

Hölldobler B. 1983. Territorial behavior in the green tree ant (Oecophyllu smaragdina). Biotropica. 15: 241-250.

Hölldobler B. \& Lumsden C. J. 1980. Territorial strategies in ants. Science. 210: 732-739.

Hölldobler B. \& Wilson E. O. 1990. The ants. Harvard University Press, Cambridge, Estados Unidos.

Hölldobler B. \& Wilson E. O. 2008. The superorganism: the beauty, elegance, and strangeness of insect societies. Norton. NewYork, 522 pp.

Hölldobler B. \& Wilson E. O. 2009. The superorganism: the beauty, elegance, and strangeness of insect societies. New York: Norton \& Co. Incorporated.

Holway D. A. 1999. Competitive mechanism underlying the displacement of native ants by the invasive Argentine Ant. Ecology. 80: 238-251.

Howard R. W., McDaniel C. A., Nelson D. R., Blomquist G. J., Gelbaum L. T. \& Zalkow L. H. 1982. Cuticular hydrocarbons of Reticulitermes virginicus (Banks) and their role as potential species- and caste-recognition cues. Journal of Chemical Ecology. 8(9): 12271239.

Hughes N. K., Price C. J. \& Banks P. B. 2010. Predators are attracted to the olfactory signals of prey. PLoS One. 5(9): 5-8.

Hunter J. S., Durant S. M. \& Caro T. M. 2007. To flee or not to flee: predator avoidance by cheetahs at kills. Behavioural Ecology and Sociobiology. 61: 1033-1042.

Ings T. C., Montoya J. M., Bascompte J., Blüthgen N., Brown L., Dormann C. F., Edwards F., Figueroa D., Jacob U., Jones J. I., Lauridsen R. B., Ledger M. E., Lewis H. M., Olesen 
J. M., van Veen F. J., Warren P. H. \& Woodward G. 2009. Ecological networks - beyond food webs. Journal of Animal Ecology. 78: 253-269.

Janzen D. H. 1966. Coevolution of mutualism between ants and acacias in Central America. Evolution. 20: 249-275.

Jordano P. 1987. Patterns of mutualistic interactions in pollination and seed dispersal: connectance, dependence asymmetries, and coevolution. American Naturalist. 129: 657677.

Jordan R. \& Blüthgen N. 2007. No evidence for a dominance-discovery trade-off among Formica pratensis colonies (Hymenoptera: Formicidae). Myrmecological News. 10: 7-10.

Kaspari M. 2000. A primer on Ant Ecology. In D. Agosti et al., (Ed.), Ants standard methods for measuring and monitoring biodiversity (pp. 9-24). Smithsonian Institution: Princeton Editorial.

Kats L. B. \& Dill L. M. 1998. The scent of death: chemosensory assessment of predation risk by prey animals. Ecoscience. 5(3): 361-394.

Kneital J. M. \& Chase J. M. 2004. Trade-offs in community ecology: linking spatial scales and species coexistence. Ecology Letters. 7: 69-80.

Lang C. \& Menzel F. 2011. Lasius niger ants discriminate aphids based on their cuticular hydrocarbons. Animal Behaviour. 82(6): 1245-1254.

Lange D., Dáttilo W. \& Del-Claro K. 2013. Influence of extrafloral nectary phenology on ant-plant mutualistic networks in a Neotropical Savanna. Ecological Entomology. 38: 463469.

Lavelle P \& Kohlmann B. 1984. Etude quantitative de la macrofaune du sol dans une foret tropicale humide du Mexique (Bonampak, Chiapas). Pedobiología. 27: 377-393.

LeBrun E. G. 2005. Who is the top dog in ant communities? Resources, parasitoids, and multiple competitive hierarchies. Oecologia. 142: $643-652$.

Lobry de Bruyn L. A. \& Conacher A. J. 1990. The role of termites and ants in soil modification: a review. Australian Journal of Soil Research Austr. 28: 55-93.

López F., Martínez M. D \& Barandica J. M. 1994. Four new species of the genus Leptanilla (Hymenoptera: Formicidae) from Spain-relationships to other species and ecological issues. Sociobiology. 24: 179-212. 
Medan D., Perazzo R. P. J., Devoto M., Burgos E., Zimmermann M. G., Ceva H. \& Delbue A. M. 2007. Analysis and assembling of network structure in mutualistic systems. Journal of Theoretical Biology. 246: 510-521.

Memmott J., Craze P. G., Waser N. M. \& Price M. V. 2007. Global warming and the disruption of plant-pollinator interactions. Ecology Letters. 10: 710-717.

Newman M. E. J. 2004. Analysis of weighted networks. Physical Review E. 70: 056131.

Newman M. E. J., Barabasi A. L. \& Watts D. J. 2006. The Structure and Dynamics of Networks. Princeton, NJ: Princeton Univ. Press.

Nieh J. C., Barreto L. S., Contrera F. A. L. \& Imperatriz-Fonseca V. L. 2004. Olfactory eavesdropping by a competitively foraging stingless bee, Trigona spinipes. Proceedings of the Royal Society B: Biological Sciences. 271(1548): 1633-1640.

Olesen J. M. \& Jordano P. 2002. Geographic patterns in plant- pollinator mutualistic networks. Ecology. 83: 2416-2424.

Parr C. L. \& Gibb H. 2010. Competition and the role of dominant ants. Ant Ecology (eds. L. Lach, C. L. Parr \& K. L. Abbott). pp. 77-96, Oxford University Press, Oxford.

Parr C. L. \& Gibb H. 2012. The discovery-dominance trade-off is the exception, rather than the rule. Journal of Animal Ecology. 81: 233-241.

Parr C. L., Sinclair B. J., Andersen A. N., Gaston K. J. \& Chown S. L. 2005. Constraint and competition in assemblages: a cross-continental and modeling approach for ants. American Naturalist. 165: 481-494.

Pearce-Duvet, J.M.C.\&Feener Jr, D.H. (2010) Resource discovery in ant communities: do food type and quantity matter? Ecological Entomology, 35, 549-556.

Pires M. M., Guimarães P. R., Araújo M. S., Giaretta A. A., Costa J. C. L. \& Dos Reis S. F. 2011. The nested assembly of individual-resource networks. Journal of Animal Ecology. 80: 896-903.

Retana J. \& Cerdá X. 2000. Patterns of diversity and composition of Mediterranean ground ant communities tracking spatial and temporal variability in the thermal environment. Oecologia. 123: $436-444$.

Rezende E. L., Jordano P. \& Bascompte J. 2007. Effects of phenotypic complementarity and phylogeny on the nested structure of mutualistic networks. Oikos. 116: 1919-1929.

Rico-Gray V. \& Oliveira P. S. 2007. The ecology and evolution of ant-plant interactions. The University of Chicago Press, Chicago, USA. 
Rico-Gray V., Díaz-Castelazo C., Ramírez-Hernández A., Guimarães P. R. \& Holland J. N. 2012. Abiotic factors shape temporal variation in the structure of an ant-plant network. Arthropod-Plant Interactions. 6: 289-295.

Sánchez-Galván I. R., Díaz-Castelazo C. \& Rico-Gray V. 2012. Effect of Hurricane Karl on a plant-ant network occurring in coastal Veracruz, Mexico. Journal of Tropical Ecology. 28: 603-609.

Santamaría L. \& Rodríguez-Gironés M. A. 2007. Linkage rules for plant-pollinator networks: trait complementarity or exploitation barriers? PLoS Biology. 5: 354-359.

Savolainen R. 1990. Colony success of the submissive ant Formica fusca within territories of the dominant Formica polyctena. Ecological Entomology. 15: 1-10.

Savolainen R. \& Vepsäläinen K. 1988. A competition hierarchy among boreal ants: impact on resource partitioning and community structure. Oikos. 51: 135-155.

Svanbäck R. \& Bolnick D. I. 2005. Intraspecific competition affects the strength of individual specialization: an optimal diet theory model. Evolutionary Ecology Research. 7: 993-1012.

Thompson J. N. 2006. Mutualistic webs of species. Science. 312: 372-373.

Tinker M. T., Guimarães P. R., Novak M., Marquitti F. M. D., Bodkin J. L., Staedler M., Bentall G. \& Estes J. A. 2012. Structure and mechanism of diet specialisation: testing models of individual variation in resource use with sea otters. Ecology Letters. 15: 475-483.

Wagner D. \& Nicklen E. F. 2010. Ant nest location, soil nutrients and nutrient uptake by ant-associated plants: does extrafloral nectar attract ant nests and thereby enhance plant nutrition? Journal of Ecology. 98: 614-624.

Wilson E. O. 1971. The Insects Societies. The Belknap Press of Harvard University Press. 548 pp. 